\title{
Diet quality is positively associated with $100 \%$ fruit juice consumption in children and adults in the United States: NHANES 2003-2006
}

\author{
Carol E O'Neil ${ }^{{ }^{* \dagger}}$, Theresa A Nicklas ${ }^{2 \dagger}$, Michael Zanovec ${ }^{1 \dagger}$, Victor L Fulgoni $\mathrm{II}^{3+}$
}

\begin{abstract}
Background: One hundred percent fruit juice (100\% FJ) has been viewed by some as a sweetened beverage with concerns about its effect on weight. Little regard has been given to the contribution of $100 \% \mathrm{FJ}$ to diet quality.

Methods: In this study data from the 2003-2006 National Health and Nutrition Examination Survey were used to examine the association of $100 \%$ FJ consumption with diet quality in participants $2-5$ years of age $(y)(n=1665)$, 6-12 y $(n=2446), 13-18$ y $(n=3139)$, and 19+y $(n=8861)$. Two 24-hour dietary recalls were used to determine usual intake using the National Cancer Institute method. Usual intake, standard errors, and regression analyses (juice independent variable and Healthy Eating Index-2005 [HEI-2005] components were dependent variables), using appropriate covariates, were determined using sample weights.
\end{abstract}

Results: The percentage of participants 2-5 y, 6-12 y, 13-18y, and 19+y that consumed 100\% FJ was 71\%, 57\%, 45\%, and $62 \%$, respectively. Usual intake of $100 \% \mathrm{FJ}$ (ounce [oz]/day) among the four age groups was: $5.8 \pm 0.6,2.6 \pm 0.4$, $3.7 \pm 0.4$, and $2.4 \pm 0.2$ for those in age groups $2-5 y, 6-12 y, 13-18 y$, and 19+y, respectively. Consumption of 100\% FJ was associated with higher energy intake in 6-12 y, 13-18 y, and 19+y; and higher total, saturated, and discretionary fats in 13-18 y participants. Consumption of 100\% FJ was associated with higher total HEl-2005 scores in all age groups (<0.0001). In 100\% FJ consumers, total and whole fruit consumption was higher and intake of added sugars was lower in all age groups.

Conclusions: Usual intake of 100\% FJ consumption exceeded MyPyramid recommendations for children 2-5 y, but was associated with better diet quality in all age groups and should be encouraged in moderation as part of a healthy diet.

\section{Background}

Consumption of fruit is associated with a variety of health benefits including an improved profile of cardiovascular disease markers [1], and a reduced risk of hypertension [2] and some types of cancer [3]. Despite these health benefits, few Americans consume the recommended amounts of fruit per day [4-6]. The MyPyramid recommendations for fruit consumption are age, gender, and physical activity specific [7]. Recommendations range from 1 cup/day for children 2-3 years of age (y) to a maximum of 2.5 cups/day for physically

\footnotetext{
* Correspondence: coneil1@lsu.edu

† Contributed equally

'School of Human Ecology, Louisiana State University Agricultural Center,

261 Knapp Hall, Baton Rouge, LA 70803, USA

Full list of author information is available at the end of the article
}

active young males. The fruit requirement can be met by consuming whole fruit-fresh, frozen, or dried, or $100 \%$ fruit juice (FJ); although the Dietary Guidelines Advisory Committee (DGAC) recommended that only $1 / 3$ of the recommendation should be met through $100 \%$ FJ to encourage fiber intake [8]. The American Academy of Pediatrics (AAP) recommends that for children 1 to $6 \mathrm{y}, 100 \%$ juice should be limited to 4 to $6 \mathrm{oz} /$ day, and for older children/adolescents, 7 to $18 \mathrm{y}$, to two 6-oz servings of juice per day [9].

Recommendations for consumption of $100 \%$ FJ by children, however, continue to be debated due to concerns about consumption and a potential link with overweight/obesity. Although some studies have shown an association between $100 \% \mathrm{FJ}$ consumption and weight [10-13], the majority have not [14-20]. A systematic

\section{() Biomed Central}


review published in 2008 showed no consistent association between consumption of $100 \%$ FJ and overweight/ obesity in children or adolescents [20]. Studies of weight and $100 \% \mathrm{FJ}$ consumption have been primarily in children since they are the principal consumers. Few studies have looked at $100 \%$ FJ consumption and weight in adults [21-23], but there is less concern than in children.

What is often overlooked in the on-going debate about $100 \% \mathrm{FJ}$ and weight and the uncertainty of how much, if any, should be consumed, is the nutrient contribution of $100 \% \mathrm{FJ}$ to the diet. One hundred percent fruit juices are nutrient dense [24] and are low in total fat, SFA, and sodium. One hundred percent fruit juices, especially grape, cranberry, pineapple, and orange juices, are high in phytochemicals [25]. They also contain a wide array of micronutrients, including vitamins A (particularly in the form of beta-carotene) and C, folate, potassium, and magnesium. The 2010 DGAC recognized vitamins $\mathrm{A}, \mathrm{C}, \mathrm{D}$, and $\mathrm{E}$; phosphorus; magnesium; potassium; and dietary fiber [26] as shortfall nutrients, and in addition, potassium and dietary fiber were nutrients of public health concern. Calcium was also a nutrient of public health concern for children and adolescents 9 to $18 \mathrm{y}$, and possibly for younger children aged 4 to $8 \mathrm{y}$, as well as for adults [26]. For adolescents (and women) of childbearing potential, folate was also identified as a nutrient of special concern [26].

Previous studies of children 2-11 y [15] and adolescents $12-18$ y [16] found that $100 \%$ FJ consumers had better nutrient intakes and higher intakes of whole fruit than those that did not consume 100\% FJ. Although the association between 100\% FJ intake and nutrient intake has been established in nationally representative samples, assessment and comparison of diet quality of $100 \%$ FJ consumers versus non-consumers is lacking. The purpose of this study was to compare the diet quality of $100 \% \mathrm{FJ}$ consumers with non-consumers in a nationally representative population.

\section{Methods}

\section{Data collection}

The National Health and Nutrition Examination Survey (NHANES) is conducted on a continual basis by the National Center for Health Statistics of the Centers for Disease Control and Prevention. One of the major objectives of NHANES is to examine the relationship between diet, nutrition, and health [27]. Details regarding the survey design, content, operations and procedures are available online [28].

\section{Study population and dietary intake}

Participants were $2 \mathrm{y}$ and older $(\mathrm{n}=16111)$ from the 2003-2006 NHANES. The study population was divided into four age groups and then further dichotomized as consumers and non-consumers of $100 \% \mathrm{FJ}$ as follows: children $2-5$ y ( $\mathrm{n}=1184$ and 481 , consumers and nonconsumers, respectively); 6-12 y ( $\mathrm{n}=1395$ and 1051); adolescents 13-18 y ( $\mathrm{n}=1397$ and 1742); and adults19+ y $(n=3394$ and 5467). Dietary data were obtained from two 24-hour dietary recalls administered using an automated multiple-pass method $[29,30]$. The first was obtained at the original interview (Day 1) and the second (Day 2) was obtained several days later via telephone. Parents/guardians provided the 24-hour dietary recalls of children 2-5 y; children 6-11 y were assisted by an adult; all others provided their own recalls. Only recall data deemed complete and reliable by the USDA Food Surveys Research Group were included in the analyses. Pregnant or lactating females $(n=711)$ were excluded from the sample. Detailed descriptions of the dietary interview methods are provided in the NHANES MEC In-Person Dietary Interviewers Procedures Manual, which includes pictures of the Computer-Assisted Dietary Interview system screens, measurement guides, and charts used to collect dietary information [31]. Due to the nature of the analysis (secondary data analysis), and the lack of personal identifiers, this study was exempted by the Institutional Review Board of the Louisiana State University Agricultural Center.

\section{Determination of $100 \%$ FJ and nutrient intake}

Two survey-specific food composition databases were used to determine the foods consumed by NHANES participants. The USDA Food and Nutrient Database for Dietary Studies (FNDDS) v. 2.0 [32] was used to determine the nutrient content of foods in 2003-2004 NHANES survey foods, and the FNDDS v. 3.0 [33] was used to determine the nutrient content of foods contained in 2005-2006 NHANES survey foods.

In this study, $100 \%$ FJ was defined according to the Federal Food and Drug Administration [34], which means that the juice was squeezed directly from fruit and that the words " $100 \%$ juice" were included on the label. Products reconstituted from concentrate with water were also considered $100 \% \mathrm{FJ}$, although their label must have included the words "reconstituted" or "made from concentrate." Juice cocktails, juice punches, juice drinks, or juice beverages were not considered as fruit juice in this study, although they contain some juice [34]. Participants were dichotomized into consumers and non-consumers of $100 \%$ FJ. Further, the amount of $100 \%$ FJ consumed was compared with the MyPyramid recommendation that $100 \%$ FJ should not account for more than $1 / 3$ of total fruit intake per day, which is equivalent to the following amounts in cups (c) per total daily energy levels: $0.33 \mathrm{c} / 1000-1200 \mathrm{kcal} ; 0.495 \mathrm{c} / 1400-$ $1800 \mathrm{kcal} ; 0.66$ c/2000-2600 kcal; and 0.825 c/2800-3200 kcal [7]. 


\section{Healthy eating index}

The Healthy Eating Index (HEI-2005) was used to determine diet quality [35]. The HEI-2005 contains 12 food components that reflect the recommendations of the 2005 Dietary Guidelines for Americans. Dietary intake is expressed per 1000 kilocalories for all components except SFA and sodium, which are fixed recommendations. The maximum possible score on the index is 100 . The first six components (i.e., total fruit; whole fruit; total vegetable; dark green, orange vegetable and legumes; total grain; and whole grain) were scored from 0 to 5 points. The next five components (i.e., milk, meat and beans, oil, SFA, and sodium) were scored from 0 to 10 points; and the last component of solid fats, alcoholic beverages, and added sugars (SoFAAS) were scored from 0 to 20 points. Scores were calculated proportionally, except for SFA and sodium; for these components, scores were prorated linearly between 0 to 8 and 8 to 10 points ( 8 and 10 points represented acceptable and optimal levels, respectively) [36]. Data files used to calculate HEI-2005 scores were downloaded from the USDA Center for Nutrition Policy and Promotion website [37].

\section{Statistical analyses}

The National Cancer Institute (NCI) method was used to estimate usual intake of selected nutrients and for HEI-2005 total scores and subcomponent scores. The two days of intake, using first day sampling weights, were used to obtain necessary variance estimates. The NCI SAS (SAS Institute, Inc., Cary, NC) macros Mixtran v.1.1 and Distrib v.1.1 were used to generate parameter estimates after covariate adjustment and to estimate the distribution of usual intake via the Monte Carlo method, respectively. Covariates for these analyses were sequence of participant's intake (Day 1 or Day 2) and a variable for weekday/weekend consumption. Differences among $100 \%$ FJ consumers and non-consumers were determined by computing population $\mathrm{Z}$ statistics generated from usual intake variables.

Regression analyses were conducted using the single 24-hour recall data (Day 1) to determine the contribution of nutrients per ounce of $100 \%$ FJ consumption. Covariates for the regression analyses were age, gender, race-ethnicity, and socioeconomic status (using poverty income ratio). Differences in beta coefficients were determined and $\mathrm{p}<0.05$ was deemed significant. For all analyses study-specific dietary fouryear sample weights [38] were used to adjust the variance for the complex sample design of NHANES using the statistical package SUDAAN (version 9.0.3 [2007] Research Triangle Institute, Research Triangle Park, NC).

\section{Results}

\section{Consumption of $100 \%$ fruit juice}

The percentage of individuals consuming 100\% FJ varied by age group, with $71 \%, 57 \%, 45 \%$, and $62 \%$ of children 2-5 y, 6-12 y, 13-18 y, and adults $19+$ y, respectively. Per capita usual intake of $100 \% \mathrm{FJ}$ (oz) among the four age groups was: $5.8 \pm 0.6,2.6 \pm 0.4,3.7 \pm 0.4$, and $2.4 \pm$ 0.2 for those $2-5 \mathrm{y}, 6-12 \mathrm{y}, 13-18 \mathrm{y}$, and $19+\mathrm{y}$, respectively. Usual intake of $100 \% \mathrm{FJ}$ (oz) among only those consuming $100 \% \mathrm{FJ}$ was higher than per capita intake: $8.5 \pm 0.8,5.4 \pm 0.8,10.0 \pm 1.0$, and $7.1 \pm 0.5$ for those 2-5 y, 6-12 y, 13-18 y, and 19+ y, respectively (data not shown).

\section{Intake of energy, fiber, added sugars, and fat}

Usual energy intake (kcal) of 100\% FJ consumers was $1657.6 \pm 47.2,2079.5 \pm 52.8,2718.0 \pm 77.5$, and $2348.7 \pm$ 47.1; for non-consumers energy intake was $1627.5 \pm 72.8$, $2089.0 \pm 66.9,2413.5 \pm 65.1,2297.0 \pm 29.7$ for age groups 2-5 y, 6-12 y, 13-18 y, and 19+ y, respectively (Table 1). Fiber intake $(\mathrm{g})$ of $100 \%$ FJ consumers was $10.3 \pm 0.4$, $12.9 \pm 0.4,15.9 \pm 0.8$, and $16.5 \pm 0.4$; for non-consumers intake was $10.2 \pm 0.7,12.9 \pm 0.6,13.2 \pm 0.6$, and $15.1 \pm$ 0.4 for age groups 2-5 y, 6-12 y, 13-18 y, and 19+y, respectively. Usual intake of added sugars (tsp) by $100 \%$ FJ consumers was $15.3 \pm 0.6,24.1 \pm 1.1,29.5 \pm 1.3$, and $20.4 \pm 0.8$; for non-consumers added sugars intake was $18.7 \pm 1.2,27.2 \pm 1.8,32.3 \pm 1.3,23.1 \pm 0.6$ for age groups 2-5 y, 6-12 y, 13-18 y, and 19+y, respectively. Usual intake of total fat (g) by $100 \%$ FJ consumers was $56.5 \pm$ $2.2,75.9 \pm 3.2,103.3 \pm 3.7$, and $87.9 \pm 1.9$; for non-consumers total fat intake was $63.5 \pm 3.7,78.8 \pm 2.8,91.8 \pm 2.8$, $88.5 \pm 1.9$ for age groups 2-5 y, 6-12 y, 13-18 y, and 19+ $\mathrm{y}$, respectively. Usual intake of SFA (g) by $100 \%$ FJ consumers was $20.9 \pm 0.9,26.9 \pm 1.2,35.9 \pm 1.4$, and $28.9 \pm$ 0.7; for non-consumers SFA intake was $23.6 \pm 1.6,27.9 \pm$ $1.0,31.4 \pm 0.9,29.3 \pm 0.7$ for age groups $2-5 \mathrm{y}, 6-12 \mathrm{y}, 13-$ $18 \mathrm{y}$, and $19+\mathrm{y}$, respectively. Usual intake of discretionary fat (g) by $100 \%$ FJ consumers was $48.0 \pm 2.0$, 64.4 \pm $2.9,86.3 \pm 3.2,69.9 \pm 1.5$; for non-consumers discretionary fat intake was $54.9 \pm 3.9,68.0 \pm 2.6,76.7 \pm 2.2,71.2 \pm$ 1.6 for age groups 2-5 y, 6-12 y, 13-18 y, and 19+y, respectively (Table 1 ).

In all age groups, except those 2-5 y, consumption of $100 \% \mathrm{FJ}$ was associated with significantly higher intakes of energy: in 6-12 y $(80.9 \pm 31.6 \mathrm{kcal} ; \mathrm{p}=0.0105)$, in 1318 y $(252.3 \pm 49.9 \mathrm{kcal} ; \mathrm{p}<0.0001)$, and in adults $19+\mathrm{y}$ $(120.9 \pm 23.7 \mathrm{kcal} ; \mathrm{p}<0.0001)$ (Table 2). Except in the youngest age groups, consumption of 100\% FJ was associated with higher intake of dietary fiber: $1.3 \pm 0.3 \mathrm{~g}$ ( $\mathrm{p}<0.0001), 2.5 \pm 0.4 \mathrm{~g}(\mathrm{p}<0.0001)$, and $1.8 \pm 1.4 \mathrm{~g}(\mathrm{p}<$ 0.0001 ), for the three other age groups, respectively. One hundred percent FJ consumption was also associated 
Table 1 Distribution of usual intake (UI) for total energy and select nutrients among $100 \%$ FJ consumers and non-consumers by age group: NHANES 2003-2006

\begin{tabular}{|c|c|c|c|c|c|c|c|c|c|}
\hline \multirow[b]{2}{*}{ Consumers/Non-consumers } & \multirow[b]{2}{*}{ Age group (y) } & \multirow[b]{2}{*}{$\mathbf{n}$} & \multirow[b]{2}{*}{ UI } & \multirow[b]{2}{*}{ SE } & \multicolumn{5}{|c|}{ Percentiles } \\
\hline & & & & & 10 & 25 & 50 & 75 & 90 \\
\hline \multicolumn{10}{|l|}{ Energy (kcal) } \\
\hline \multirow[t]{4}{*}{ Consumers } & $2-5 y$ & 1184 & 1657.6 & 47.2 & 1225.8 & 1407.3 & 1629.5 & 1880.0 & 2124.3 \\
\hline & $6-12 y$ & 1395 & 2079.5 & 52.8 & 1582.1 & 1797.8 & 2054.1 & 2333.5 & 2606.1 \\
\hline & $13-18 y$ & 1397 & 2718.0 & 77.5 & 1740.5 & 2121.7 & 2616.1 & 3200.0 & 3825.1 \\
\hline & $19+y$ & 3394 & 2348.7 & 47.1 & 1488.8 & 1826.5 & 2266.8 & 2780.6 & 3314.4 \\
\hline \multirow[t]{4}{*}{ Non-consumers } & $2-5 y$ & 481 & 1627.5 & 72.8 & 1159.7 & 1353.0 & 1594.6 & 1865.8 & 2137.6 \\
\hline & $6-12$ y & 1051 & 2089.0 & 66.9 & 1585.1 & 1807.6 & 2069.6 & 2349.4 & 2613.6 \\
\hline & $13-18$ y & 1742 & 2413.5 & 65.1 & 1516.3 & 1877.9 & 2337.5 & 2871.1 & 3402.8 \\
\hline & $19+y$ & 5467 & 2297.0 & 29.7 & 1411.4 & 1761.1 & 2214.9 & 2742.8 & 3288.5 \\
\hline \multicolumn{10}{|l|}{ Fiber $(g)$} \\
\hline \multirow[t]{4}{*}{ Consumers } & $2-5 y$ & 1184 & 10.3 & 0.4 & 6.6 & 8.1 & 10.0 & 12.2 & 14.4 \\
\hline & $6-12 y$ & 1395 & 12.9 & 0.4 & 8.9 & 10.5 & 12.6 & 15.0 & 17.3 \\
\hline & $13-18$ y & 1397 & 15.9 & 0.8 & 9.4 & 11.8 & 15.1 & 19.1 & 23.5 \\
\hline & $19+y$ & 3394 & 16.5 & 0.4 & 9.4 & 12.1 & 15.7 & 20.0 & 24.5 \\
\hline \multirow[t]{4}{*}{ Non-consumers } & $2-5 y$ & 481 & 10.2 & 0.7 & 6.2 & 7.8 & 9.8 & 12.1 & 14.7 \\
\hline & $6-12 y$ & 1051 & 12.9 & 0.6 & 9.6 & 11.0 & 12.7 & 14.7 & 16.6 \\
\hline & $13-18 y$ & 1742 & 13.2 & 0.6 & 7.6 & 9.8 & 12.7 & 16.1 & 19.6 \\
\hline & $19+y$ & 5467 & 15.1 & 0.4 & 8.1 & 10.8 & 14.3 & 18.6 & 23.0 \\
\hline \multicolumn{10}{|l|}{ Added sugars (tsp) } \\
\hline \multirow[t]{4}{*}{ Consumers } & $2-5 y$ & 1184 & 15.3 & 0.6 & 8.4 & 11.0 & 14.5 & 18.7 & 23.0 \\
\hline & $6-12 y$ & 1395 & 24.1 & 1.1 & 14.4 & 18.3 & 23.3 & 29.0 & 34.9 \\
\hline & $13-18$ y & 1397 & 29.5 & 1.3 & 15.4 & 20.7 & 28.0 & 36.8 & 45.8 \\
\hline & $19+y$ & 3394 & 20.4 & 0.8 & 7.4 & 11.6 & 18.1 & 26.6 & 36.4 \\
\hline \multirow[t]{4}{*}{ Non-consumers } & $2-5 y$ & 481 & 18.7 & 1.2 & 8.2 & 12.0 & 17.4 & 23.9 & 30.8 \\
\hline & $6-12 y$ & 1051 & 27.2 & 1.8 & 16.4 & 20.7 & 26.3 & 32.7 & 39.3 \\
\hline & $13-18$ y & 1742 & 32.3 & 1.3 & 15.3 & 21.7 & 30.4 & 40.7 & 51.7 \\
\hline & $19+y$ & 5467 & 23.1 & 0.6 & 7.2 & 12.2 & 19.9 & 30.6 & 43.0 \\
\hline \multicolumn{10}{|l|}{ Total fat (g) } \\
\hline \multirow[t]{4}{*}{ Consumers } & $2-5 y$ & 1184 & 56.5 & 2.2 & 37.3 & 45.0 & 55.0 & 66.3 & 77.8 \\
\hline & $6-12 y$ & 1395 & 75.9 & 3.2 & 54.3 & 63.5 & 74.8 & 87.0 & 98.8 \\
\hline & $13-18 y$ & 1397 & 103.3 & 3.7 & 61.7 & 77.8 & 99.1 & 124.4 & 150.5 \\
\hline & $19+y$ & 3394 & 87.9 & 1.9 & 51.2 & 65.4 & 84.2 & 106.3 & 129.5 \\
\hline \multirow[t]{4}{*}{ Non-consumers } & $2-5 y$ & 481 & 63.5 & 3.7 & 42.7 & 51.2 & 61.9 & 74.2 & 86.5 \\
\hline & $6-12 y$ & 1051 & 78.8 & 2.8 & 55.6 & 65.6 & 77.7 & 90.7 & 103.5 \\
\hline & $13-18$ y & 1742 & 91.8 & 2.8 & 56.2 & 70.5 & 88.8 & 109.7 & 131.1 \\
\hline & $19+y$ & 5467 & 88.5 & 1.9 & 50.1 & 65.1 & 84.7 & 107.8 & 131.7 \\
\hline \multicolumn{10}{|l|}{ SFA (g) } \\
\hline Consumers & $2-5 y$ & 1184 & 20.9 & 0.9 & 13.1 & 16.2 & 20.2 & 24.8 & 29.5 \\
\hline & $6-12 y$ & 1395 & 26.9 & 1.2 & 18.9 & 22.3 & 26.5 & 31.0 & 35.5 \\
\hline & $13-18 y$ & 1397 & 35.9 & 1.4 & 21.0 & 26.8 & 34.5 & 43.4 & 52.6 \\
\hline & $19+y$ & 3394 & 28.9 & 0.7 & 15.9 & 20.8 & 27.4 & 35.4 & 43.9 \\
\hline Non-consumers & $2-5 y$ & 481 & 23.6 & 1.6 & 15.5 & 18.8 & 23.0 & 27.7 & 32.6 \\
\hline & $6-12 y$ & 1051 & 27.9 & 1.0 & 19.4 & 23.0 & 27.5 & 32.3 & 37.1 \\
\hline & $13-18 y$ & 1742 & 31.4 & 0.9 & 18.8 & 23.8 & 30.4 & 37.9 & 45.5 \\
\hline & $19+y$ & 5467 & 29.3 & 0.7 & 15.8 & 20.9 & 27.8 & 36.1 & 44.9 \\
\hline Discretionary fat $(\mathrm{g})$ & & & & & & & & & \\
\hline Consumers & $2-5 y$ & 1184 & 48.0 & 2.0 & 31.1 & 38.0 & 46.7 & 56.5 & 66.5 \\
\hline & $6-12 y$ & 1395 & 64.4 & 2.9 & 44.6 & 53.0 & 63.2 & 74.6 & 85.6 \\
\hline & $13-18$ y & 1397 & 86.3 & 3.2 & 50.1 & 64.1 & 82.6 & 104.6 & 127.2 \\
\hline
\end{tabular}


Table 1 Distribution of usual intake (UI) for total energy and select nutrients among $100 \%$ FJ consumers and non-consumers by age group: NHANES 2003-2006 (Continued)

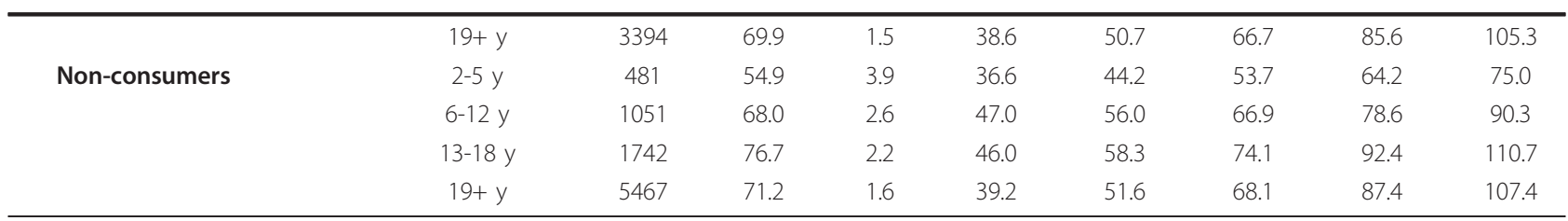

Data are presented as sample-weighted mean usual intake $\pm \mathrm{SE}$, and percentiles of usual intake. Abbreviations: kcal, kilocalories; tsp, teaspoon; g, grams; NHANES, National Health and Nutrition Examination Survey; y, years; n, number; UI, usual intake; SE, standard error; SFA, saturated fatty acids. Note: There are $4.2 \mathrm{~g}$ in one tsp of sugar.

with lower intake of added sugars (tsp) in all age groups: $-4.6 \pm 0.7(\mathrm{p}<0.0001)$ in $2-5 \mathrm{y},-2.8 \pm 0.7(\mathrm{p}<0.0001)$ in $6-12 \mathrm{y},-1.8 \pm 0.9(\mathrm{p}=0.0396)$ in $13-18 \mathrm{y}$ and $-1.0 \pm 0.4$ $(\mathrm{p}=0.0154)$ in $19+\mathrm{y}$, respectively.

Adolescents 13-18 y were the only age group in which consumers of $100 \% \mathrm{FJ}$ had a higher intake of total fat, SFA, and discretionary fat than non-consumers (Table 1).

\section{Diet quality}

The HEI-2005 scores of 100\% FJ consumers by age were $53.0 \pm 1.2,49.3 \pm 1.7,49.6 \pm 0.9$, and $52.6 \pm 0.6$, for age groups 2-5 y, 6-12 y, 13-18 y, and 19+ y, respectively (Table 3). The HEI-2005 scores of non-consumers by age were $47.3 \pm 2.1,44.1 \pm 1.7,44.4 \pm 1.0$, and $47.7 \pm$ 0.5 , for age groups 2-5 y, 6-12 y, 13-18 y, and 19+y, respectively. For all age groups, consumers of $100 \%$ FJ had higher HEI-2005 scores than non-consumers, even when HEI-2005 scores were compared across percentiles (data not shown).

Results of the regression analysis of $100 \%$ FJ consumption with total HEI-2005 and HEI-2005 subcomponent scores by age group are presented in Table 4. Consumption of $100 \% \mathrm{FJ}$ contributed to the total HEI-2005 score in each age group by $7.1 \pm 0.8,5.5 \pm 0.5,5.6 \pm 0.5$, and $5.1 \pm 0.3$ points, respectively ( $\mathrm{p}<0.0001$ for all). Total fruit scores were higher in each age group by $1.9 \pm 0.1$, $1.7 \pm 0.1,2.0 \pm 0.1$, and $1.7 \pm 0.1$, respectively $(\mathrm{p}<$
0.001 for all). Whole fruit scores were also higher in all ages groups by $0.5 \pm 0.1(\mathrm{p}=0.0006), 0.3 \pm 0.1(\mathrm{p}=$ $0.0010), 0.3 \pm 0.1(\mathrm{p}=0.0004)$, and $0.4 \pm 0.05(\mathrm{p}<$ $0.0001)$, respectively. The dark green/orange vegetables/ legumes scores were higher in children 6-12 y $(0.2 \pm 0.1$ $[\mathrm{p}=0.0007])$ and adults $19+\mathrm{y}(0.1 \pm 0.1[\mathrm{p}=0.0030])$, but not in the other age groups (data not shown). Whole grains scores were significantly higher in children $2-5$ y $(0.2 \pm 0.1[\mathrm{p}=0.0323]), 6-12$ y $(0.2 \pm 0.1[\mathrm{p}=$ $0.0011])$, and adults $19+\mathrm{y}(0.2 \pm 0.1[\mathrm{p}<0.0001])$ (data not shown). The most significant contribution to the overall HEI-2005 score was the improvement in the SoFAAS score: $3.4 \pm 0.4,2.3 \pm 0.3,2.3 \pm 0.3$, and $1.7 \pm$ 0.2 , respectively in the four age groups $(\mathrm{p}<0.0001$ for all).

The distribution of total HEI-2005 usual intake scores of consumers and non-consumers of $100 \% \mathrm{FJ}$ is shown in Table 5. In all age groups, consumers had a higher $(\mathrm{p}<0.05)$ HEI-2005 score than non-consumers: $2-5 \mathrm{y}$ $(53.0 \pm 1.2$ vs. $47.3 \pm 2.1), 6-12$ y $(49.3 \pm 1.7$ vs. $44.1 \pm$ $1.7), 13-18$ y ( $49.6 \pm 0.9$ vs. $44.4 \pm 1.0)$, and $19+\mathrm{y}(52.6 \pm$ 0.6 vs. $47.7 \pm 0.5)$.

\section{Discussion}

This study showed that the percentage of $100 \% \mathrm{FJ}$ consumers was age dependent; children $2-5$ y had the highest percentage of consumers and adolescents 13-18 y had the lowest percentage of consumers. Diet quality,

Table 2 Regression analysis of $100 \%$ FJ consumption (any amount) with total energy and select nutrients by age group: NHANES 2003-2006

\begin{tabular}{|c|c|c|c|c|c|c|c|c|c|c|c|c|}
\hline \multirow[b]{3}{*}{ Variable } & \multicolumn{12}{|c|}{ Juice consumers ( $>0 \mathrm{oz} /$ day) } \\
\hline & \multicolumn{3}{|c|}{$\begin{array}{c}2-5 y \\
(n=1184)\end{array}$} & \multicolumn{3}{|c|}{$\begin{array}{c}6-12 y \\
(n=1395)\end{array}$} & \multicolumn{3}{|c|}{$\begin{array}{c}13-18 y \\
(n=1397)\end{array}$} & \multicolumn{3}{|c|}{$\begin{array}{c}19+y \\
(n=3394)\end{array}$} \\
\hline & $\beta$ & SE & $P$-value & $\beta$ & SE & $P$-value & $\beta$ & SE & $P$-value & $\beta$ & SE & $P$-value \\
\hline Energy (kcal) & 39.6 & 35.9 & 0.2709 & 80.9 & 31.6 & 0.0105 & 252.3 & 49.9 & $<0.0001$ & 120.9 & 23.7 & $<0.0001$ \\
\hline Fiber (g) & 0.7 & 0.4 & 0.06 & 1.3 & 0.3 & $<0.0001$ & 2.5 & 0.4 & $<0.0001$ & 1.8 & 1.4 & $<0.0001$ \\
\hline Added sugars (tsp) & -4.6 & 0.7 & $<0.0001$ & -2.8 & 0.7 & $<0.0001$ & -1.8 & 0.9 & 0.0396 & -1.0 & 0.4 & 0.0154 \\
\hline Total fat (g) & -1.5 & 1.7 & 0.4026 & 0.9 & 1.5 & 0.5430 & 6.3 & 2.2 & 0.0035 & 0.8 & 1.1 & 0.4580 \\
\hline SFA (g) & -0.8 & 0.7 & 0.2371 & 0.5 & 0.6 & 0.3980 & 1.9 & 0.8 & 0.0152 & -0.15 & 0.39 & 0.7034 \\
\hline Discretionary fat (g) & -1.7 & 1.5 & 0.2367 & -0.4 & 1.4 & 0.7890 & 4.8 & 1.9 & 0.0118 & 0.1 & 0.9 & 0.9417 \\
\hline
\end{tabular}

Data are presented as unstandardized regression coefficients ( $\beta$ ), standard errors (SE), and $P$-values of $\beta$ coefficients. Abbreviations: FJ, fruit juice; $y$, years; SE, standard error; kcal, kilocalories; tsp, teaspoon; g, gram; SFA, saturated fatty acids. Note: There are $4.2 \mathrm{~g}$ in one tsp of sugar. 
Table 3 Comparison of HEl-2005 total and select subcomponent usual intake (UI) scores between $100 \% \mathrm{FJ}$ consumers and non-consumers by age group: NHANES 2003-2006

\begin{tabular}{|c|c|c|c|c|c|c|c|}
\hline \multirow[b]{2}{*}{ Variable } & \multirow[b]{2}{*}{$\begin{array}{l}\text { Age group } \\
\text { (y) }\end{array}$} & \multicolumn{3}{|c|}{ Consumers } & \multicolumn{3}{|c|}{ Non-Consumers } \\
\hline & & $\mathrm{n}$ & $\begin{array}{l}\text { UI } \\
\text { score }\end{array}$ & SE & $\mathrm{n}$ & $\begin{array}{l}\text { UI } \\
\text { score }\end{array}$ & SE \\
\hline \multirow{4}{*}{$\begin{array}{l}\text { HEl-2005-Total } \\
\text { Score }\end{array}$} & $2-5 y$ & 1184 & 53.0 & 1.2 & 481 & 47.3 & 2.1 \\
\hline & $6-12 y$ & 1395 & 49.3 & 1.7 & 1051 & 44.1 & 1.7 \\
\hline & $13-18 y$ & 1397 & 49.6 & 0.9 & 1742 & 44.4 & 1.0 \\
\hline & $19+y$ & 3394 & 52.6 & 0.6 & 5467 & 47.7 & 0.5 \\
\hline \multirow{4}{*}{$\begin{array}{l}\text { HEl-2005-Total } \\
\text { Fruit }\end{array}$} & $2-5 y$ & 1184 & 4.1 & 0.1 & 481 & 1.8 & 0.2 \\
\hline & $6-12 y$ & 1395 & 3.0 & 0.3 & 1051 & 1.4 & 0.2 \\
\hline & $13-18 y$ & 1397 & 3.2 & 0.3 & 1742 & 1.1 & 0.1 \\
\hline & $19+y$ & 3394 & 3.0 & 0.2 & 5467 & 1.3 & 0.1 \\
\hline \multirow{4}{*}{$\begin{array}{l}\text { HEl-2005-Whole } \\
\text { Fruit }\end{array}$} & $2-5 y$ & 1184 & 2.4 & 0.2 & 481 & 1.8 & 0.3 \\
\hline & $6-12 y$ & 1395 & 1.9 & 0.2 & 1051 & 1.8 & 0.3 \\
\hline & $13-18 y$ & 1397 & 1.7 & 0.7 & 1742 & 1.2 & 0.1 \\
\hline & $19+y$ & 3394 & 2.2 & 0.1 & 5467 & 1.6 & 0.1 \\
\hline \multirow[t]{4}{*}{ HEI-2005-Milk } & $2-5 y$ & 1184 & 7.8 & 0.2 & 481 & 8.2 & 0.2 \\
\hline & $6-12$ y & 1395 & 7.0 & 0.3 & 1051 & 6.4 & 0.3 \\
\hline & $13-18 y$ & 1397 & 5.9 & 0.3 & 1742 & 5.9 & 0.3 \\
\hline & $19+y$ & 3394 & 4.9 & 0.2 & 5467 & 4.6 & 0.2 \\
\hline \multirow[t]{4}{*}{ HEI-2005-SFA } & $2-5 y$ & 1184 & 5.7 & 0.6 & 481 & 4.0 & 0.8 \\
\hline & $6-12$ y & 1395 & 5.0 & 0.4 & 1051 & 5.2 & 0.5 \\
\hline & $13-18 y$ & 1397 & 5.3 & 0.3 & 1742 & 5.4 & 0.2 \\
\hline & $19+y$ & 3394 & 6.0 & 0.2 & 5467 & 5.9 & 0.2 \\
\hline \multirow[t]{4}{*}{ HEl-2005-Sodium } & $2-5 y$ & 1184 & 5.1 & 0.3 & 481 & 4.6 & 0.4 \\
\hline & $6-12 y$ & 1395 & 4.4 & 0.3 & 1051 & 4.6 & 0.4 \\
\hline & $13-18 y$ & 1397 & 4.6 & 0.3 & 1742 & 4.8 & 0.3 \\
\hline & $19+y$ & 3394 & 4.6 & 0.2 & 5467 & 4.1 & 0.1 \\
\hline \multirow[t]{4}{*}{ HEI-2005-SOFAAS } & $2-5 y$ & 1184 & 10.1 & 0.6 & 481 & 6.8 & 0.9 \\
\hline & $6-12 y$ & 1395 & 7.7 & 0.6 & 1051 & 5.4 & 0.8 \\
\hline & $13-18 y$ & 1397 & 8.1 & 0.5 & 1742 & 5.6 & 0.6 \\
\hline & $19+y$ & 3394 & 9.0 & 0.3 & 5467 & 7.6 & 0.3 \\
\hline
\end{tabular}

Abbreviations: HEl-2005, Healthy Eating Index-2005; y, years; n, number; UI, usual intake; SE, standard error; SFA, saturated fatty acids; SoFAAS, solid fats, alcoholic beverages, and added sugars.

determined by the HEI-2005, was better in all age groups of $100 \%$ FJ consumers when compared with nonconsumers.

The age variation of the percentage of $100 \%$ FJ consumers is consistent with other studies $[15,16]$. Reasons for the high prevalence of 100\% FJ consumption in children may include participation in the Women, Infants, and Children (WIC) Program by young children [39] since $100 \% \mathrm{FJ}$ is a WIC authorized food in three of the food packages for children over 11 months of age [40]. Participation in the National School Lunch or Breakfast Program by older children [41] may also lead to consumption of $100 \% \mathrm{FJ}$ since these are approved menu
Table 4 Regression analysis of $100 \%$ FJ consumption with HEI-2005 total and subcomponent scores by age group: NHANES 2003-2006

\begin{tabular}{|c|c|c|c|}
\hline \multirow[b]{2}{*}{ Variable } & \multicolumn{3}{|c|}{ Juice consumers ( $>0$ oz/day) } \\
\hline & $\beta$ & SE & $P$-value \\
\hline \multicolumn{4}{|c|}{ Total HEI-2005 } \\
\hline $2-5 y$ & 7.1 & 0.8 & $<0.0001$ \\
\hline $6-12$ y & 5.5 & 0.5 & $<0.0001$ \\
\hline $13-18$ y & 5.6 & 0.5 & $<0.0001$ \\
\hline $19+y$ & 5.1 & 0.3 & $<0.0001$ \\
\hline \multicolumn{4}{|l|}{ Total fruit } \\
\hline $2-5 y$ & 1.9 & 0.1 & $<0.0001$ \\
\hline $6-12 y$ & 1.7 & 0.1 & $<0.0001$ \\
\hline $13-18$ y & 2.0 & 0.1 & $<0.0001$ \\
\hline $19+y$ & 1.7 & 0.1 & $<0.0001$ \\
\hline \multicolumn{4}{|l|}{ Whole fruit } \\
\hline $2-5 y$ & 0.5 & 0.1 & 0.0006 \\
\hline $6-12 y$ & 0.3 & 0.1 & 0.0010 \\
\hline $13-18$ y & 0.3 & 0.1 & 0.0004 \\
\hline $19+y$ & 0.4 & 0.1 & $<0.0001$ \\
\hline \multicolumn{4}{|l|}{ Milk } \\
\hline $2-5 y$ & -0.2 & 0.2 & 0.3326 \\
\hline $6-12 y$ & 0.2 & 0.2 & 0.1881 \\
\hline $13-18$ y & -0.0 & 0.1 & 0.8766 \\
\hline $19+y$ & 0.0 & 0.1 & 0.8867 \\
\hline \multicolumn{4}{|l|}{ SFA } \\
\hline $2-5 y$ & 0.7 & 0.2 & 0.0019 \\
\hline $6-12 y$ & 0.3 & 0.2 & 0.0621 \\
\hline $13-18$ y & 0.7 & 0.1 & $<0.0001$ \\
\hline $19+y$ & 0.6 & 0.1 & $<0.0001$ \\
\hline \multicolumn{4}{|l|}{ Sodium } \\
\hline $2-5 y$ & 0.4 & 0.2 & 0.0268 \\
\hline $6-12$ y & 0.2 & 0.1 & 0.2173 \\
\hline $13-18$ y & 0.5 & 0.1 & 0.0001 \\
\hline $19+y$ & 0.4 & 0.1 & $<0.0001$ \\
\hline \multicolumn{4}{|l|}{ SoFAAS } \\
\hline $2-5 y$ & 3.4 & 0.4 & $<0.0001$ \\
\hline $6-12 y$ & 2.3 & 0.3 & $<0.0001$ \\
\hline $13-18$ y & 2.3 & 0.3 & $<0.0001$ \\
\hline $19+y$ & 1.7 & 0.2 & $<0.0001$ \\
\hline
\end{tabular}

Data are presented as unstandardized regression coefficients ( $\beta)$, standard errors (SE), and $P$-values of $\beta$ coefficients.

Abbreviations: y, years; SE, standard error; HEl-2005, Healthy Eating Index2005; SFA, saturated fatty acids; SoFAAS, solid fats, alcoholic beverages, and added sugars.

items, and is the only juice product that may be served in the National School Breakfast Program [42]. It is also possible the frequency of consumption is lowest in adolescents since they are the least likely age group to consume breakfast [43] and 100\% FJ is thought of as a breakfast food by many consumers. Adolescents who participate in the National School Lunch program also have more beverage options than elementary school children [44] have and may not choose $100 \%$ FJ. Studies 
Table 5 Distribution of HEl-2005 total usual intake (UI) scores among 100\% FJ consumers and non-consumers by age group: NHANES 2003-2006

\begin{tabular}{|c|c|c|c|c|c|c|c|c|c|}
\hline & \multirow[b]{2}{*}{ Age group (y) } & \multicolumn{3}{|c|}{ HEl-2005 } & \multicolumn{5}{|c|}{ Percentiles } \\
\hline & & $\mathrm{n}$ & UI & SE & 10 & 25 & 50 & 75 & 90 \\
\hline \multirow[t]{4}{*}{ Consumers } & $2-5 y$ & 1184 & $53.0^{\mathrm{a}}$ & 1.2 & 44.0 & 48.3 & 53.0 & 57.7 & 61.9 \\
\hline & $6-12 y$ & 1395 & $49.3^{a}$ & 1.7 & 42.3 & 45.5 & 49.2 & 52.9 & 56.3 \\
\hline & $13-18$ y & 1397 & $49.6^{a}$ & 0.9 & 41.2 & 45.0 & 49.5 & 53.9 & 58.0 \\
\hline & $19+y$ & 3394 & $52.6^{a}$ & 0.6 & 41.5 & 46.7 & 52.5 & 58.5 & 63.8 \\
\hline \multirow[t]{4}{*}{ Non-consumers } & $2-5 y$ & 481 & $47.3^{b}$ & 2.1 & 38.8 & 42.5 & 47.0 & 51.7 & 56.4 \\
\hline & $6-12 y$ & 1051 & $44.1^{b}$ & 1.7 & 37.2 & 40.3 & 43.9 & 47.7 & 51.2 \\
\hline & $13-18$ y & 1742 & $44.4^{\mathrm{b}}$ & 1.0 & 36.6 & 40.1 & 44.1 & 48.5 & 52.5 \\
\hline & $19+y$ & 5467 & $47.7^{b}$ & 0.5 & 36.4 & 41.5 & 47.4 & 53.5 & 59.4 \\
\hline
\end{tabular}

${ }^{\mathrm{ab}}$ Usual HEI-2005 means with different superscripts within age groups are significantly $(p<0.05)$ different. Abbreviations: $y$, years; $n$, number; UI, usual intake; SE, standard error.

have also shown a secular decline in consumption of $100 \%$ FJ by adolescents over the past five years [45].

MyPyramid [7] and AAP [9] recommendations state that $100 \% \mathrm{FJ}$ can be part of a healthy diet when served in age appropriate amounts. This study showed that the usual intake for children 2-5 y exceeded these recommendations. Further studies are needed to assess the health effects of consuming higher than recommended levels of $100 \% \mathrm{FJ}$, and whether consuming whole fruit without $100 \%$ FJ provides an advantage to consumers. Recommendations for $100 \%$ FJ consumption vary; thus, caretakers and consumers may be confused and efforts should be made to reconcile the recommendations using an evidence-based approach.

MyPyramid recommendations were established, in part, to encourage fiber intake since modeling studies by the USDA showed that when $100 \%$ FJ was replaced by whole fruit, fiber intake increased by nearly $37 \%$ [8]. Regression analysis did show a modest, but significant contribution of fiber to the diet from 100\% FJ in all but the youngest age group. That there was no difference in fiber intake between 100\% FJ consumers and nonconsumers suggests that other foods were contributing fiber to the diet. Usual fiber intake was, however, below the Institute of Medicine's recommendations [46] in all age groups in $100 \% \mathrm{FJ}$ consumers and non-consumers and foods rich in fiber should be encouraged.

The rationale for the AAP recommendations for 100\% FJ consumption in children and adolescents is not completely clear, but is in part based on one study that showed an association of $100 \% \mathrm{FJ}$ consumption and overweight in pre-school aged children [10]. Although concerns about overweight/obesity and consumption of $100 \%$ FJ appear unfounded [20], it is important to consume $100 \% \mathrm{FJ}$ with other foods while maintaining energy balance. Paradoxically, in all age groups except children $2-5 \mathrm{y}$, the group that consumed the most $100 \% \mathrm{FJ}$, energy was higher in $100 \% \mathrm{FJ}$ consumers. The relationship of weight and $100 \% \mathrm{FJ}$ consumption was not examined in this study.

Previous studies have shown that children [15] and adolescents [16] also had higher intakes of total fruit and adolescents had higher intakes of citrus, melons, and berries. These studies have not been conducted in adults. Moreover, studies have not examined the effect of $100 \%$ FJ intake on overall diet quality. The HEI-2005, used to assess diet quality, was revised to reflect the 2005 Dietary Guidelines [47]. The HEI-2005 now reflects all components of the MyPyramid eating plan, including grains/ whole grains, fruit/fruit juice, variety in vegetable and fat types, non/low fat dairy, sodium, and discretionary calories [35]. Traditionally, population HEI-2005 scores have been used with a single 24-hour dietary recall [48], but recently it was shown that it could also be used when multiple recalls were available [49].

The HEI-2005 scores for this population were lower than those shown for individuals $2+\mathrm{y}$ in 2003-2004 [50]. However, the overall scores of those consuming $100 \%$ FJ were significantly higher than those not consuming juice. Consumption of $100 \% \mathrm{FJ}$ improved total HEI-2005 scores in all age groups; by choosing to consume this single food, the HEI-2005 score improved approximately $10 \%$. However, the total HEI-2005 scores calculated in this study both with and without $100 \%$ FJ consumption were still low and needed improvement.

Consumers of $100 \% \mathrm{FJ}$ also had improved intake of whole fruit. When assessing consumption levels, many studies have combined the intake of fruit and vegetables; however, those studies reporting fruit separately have shown that children, adolescents, or adults [4-6,51] did not meet the fruit recommendation. Data from the 2003-2004. NHANES showed that adult females had the highest percentage $(12.3 \%)$ of those meeting the fruit recommendation; only $8.6 \%$ of adult males met the recommendation [4]. For both genders, the median 
number of servings consumed was 0.61 per day [4]. Overall consumption was lowest in pre-adolescent, adolescent, and young adult males [5]. Despite extensive, coordinated public health campaigns by government and industry [52], fruit consumption remains low. Consumption of $100 \% \mathrm{FJ}$ appears to be one way to increase fruit intake; however, the goal should be to encourage intake of all types of fruit.

One concern about consumption of $100 \%$ FJ in children and adolescents is that it may replace fluid milk in the diet [53]. Regression analyses showed that there was no difference in the HEI-2005 Milk component score between consumers and non-consumers of 100\% FJ. This supports findings from previous studies that consumption of $100 \% \mathrm{FJ}$ was not associated with lower consumption of milk in the diets of children [15,16].

This study did show that in adolescents 13-18 y consumption of $100 \%$ FJ was also associated with higher intake of total, saturated, and discretionary fats. Reasons for this are not clear, but may reflect the overall poor eating habits reported in this age group [43,54]. Additional studies are needed to determine factors leading to poor diet quality in adolescents.

\section{Limitations}

NHANES is a cross-sectional study, thus cause and effect relationships cannot be determined. Participants relied on memory to self-report dietary intakes; therefore, data were subject to non-sampling errors, including under- or over-reporting of energy. Parents reported or assisted their children 2-11 y with the 24-hour recalls; parents often report accurately what children eat in the home [55] but may not know what their children consume outside the home [56], which could also result in reporting errors [57].

\section{Conclusions}

This study showed that, with the exception of children 2-5 y, usual intake of $100 \% \mathrm{FJ}$ was within the MyPyramid recommendations for children, adolescents, and adults, and the AAP recommendations for children and adolescents. Among consumers, all age groups exceeded MyPyramid recommendations for $100 \%$ FJ consumption. Consumption of any amount of $100 \%$ FJ was associated with improved diet quality in all age groups. Due to its contribution to overall diet quality, 100\% FJ should be recommended to all age groups as a component of a healthy diet.

\section{Abbreviations}

AAP: American Academy of Pediatrics; FNDDS: Food and Nutrient Database for Dietary Studies; HEl-2005: Healthy Eating Index - 2005; FJ: fruit juice; DGAC: Dietary Guidelines Advisory Committee; MEC: Mobile Examination Center; NHANES: National Health and Nutrition Examination Survey; SoFAAS:
Solid fats, alcoholic beverages, and added sugars; USDA: United States Department of Agriculture; WIC: Women, Infants, and Children.

\section{Acknowledgements}

This work is a publication of the United States Department of Agriculture (USDA/ARS) Children's Nutrition Research Center, Department of Pediatrics, Baylor College of Medicine, and Houston, Texas. The contents of this publication do not necessarily reflect the views or policies of the USDA, nor does mention of trade names, commercial products, or organizations imply endorsement from the U.S. government. This research project was supported by the USDA - Agricultural Research Service through specific cooperative agreement 58-6250-6-003 and the Juice Products Association Partial support was received from the USDA Hatch Project LAB 93951. Other than the partial funding of this study from the Juice Products Association, the sponsor had no role in the design and conduction of the study; the collection, management, analysis, and interpretation of the data; or the preparation and approval of the manuscript

\section{Author details}

${ }^{1}$ School of Human Ecology, Louisiana State University Agricultural Center, 261 Knapp Hall, Baton Rouge, LA 70803, USA. USDA/ARS Children's Nutrition Research Center, Department of Pediatrics, Baylor College of Medicine, 1100 Bates Avenue, Houston, TX 77030, USA. ${ }^{3}$ Nutrition Impact, LLC, 9725 D Drive North, Battle Creek, MI 49014, USA

\section{Authors' contributions}

CEO, TAN, and VLF designed this study and had full access to all the data and they take responsibility for the integrity of the data and the accuracy of the data analysis. MZ had full access to all data, worked with collating the data, and with data re-analyses. CEO and TAN were the principal authors. All authors read and approved the final manuscript.

\section{Competing interests}

The authors declare that they have no competing interests.

Received: 17 November 2010 Accepted: 13 February 2011 Published: 13 February 2011

\section{References}

1. Basu A, Penugonda K: Pomegranate juice: a heart-healthy fruit juice. Nutr Rev 2009, 67:49-56.

2. Tsubota-Utsugi M, Ohkubo T, Kikuya M, Metoki H, Kurimoto A, Suzuki K, Fukushima N, Hara A, Asayama K, Satoh H, Tsubono Y, Imai Y: High fruit intake is associated with a lower risk of future hypertension determined by home blood pressure measurement: the OHASAMA study. J Hum Hypertens 2011, 25:164-171.

3. Boffetta P, Couto E, Wichmann J, Ferrari P, Trichopoulos D, Bueno-deMesquita HB, van Duijnhoven FJ, Buchner FL, Key T, Boeing $H$, Nothlings $U$, Linseisen J, Gonzalez CA, Overvad K, Nielsen MR, Tjonneland A, Olsen A, Clavel-Chapelon F, Boutron-Ruault MC, Morois S, Lagiou P, Naska A, Benetou V, Kaaks R, Rohrmann S, Panico S, Sieri S, Vineis P, Palli D, van Gils $\mathrm{CH}$, et al: Fruit and vegetable intake and overall cancer risk in the European Prospective Investigation into Cancer and Nutrition (EPIC). J Natl Cancer Inst 2010, 102:529-37.

4. Kimmons J, Gillespie C, Seymour J, Serdula M, Blanck HM: Fruit and vegetable intake among adolescents and adults in the United States: percentage meeting individualized recommendations. Medscape J Med 2009, 11:26.

5. Guenther PM, Dodd KW, Reedy J, Krebs-Smith SM: Most Americans eat much less than recommended amounts of fruits and vegetables. J Am Diet Assoc 2006, 106:1371-9.

6. Blanck HM, Gillespie C, Kimmons JE, Seymour JD, Serdula MK: Trends in fruit and vegetable consumption among U.S. men and women, 19942005. Prev Chronic Dis 2008, 5:A35.

7. U.S. Department of Agriculture: MyPyramid Web Page [http://www mypyramid.gov]

8. U.S. Department of Agriculture, Dietary Guidelines Advisory Committee, Agricultural Research Service: Report of the Dietary Guidelines Advisory Committee on the Dietary Guidelines for Americans 2005. Washington DC 2005 [http://www.health.gov/dietaryguidelines/dga2005/report]. 
9. American Academy of Pediatrics, Committee on Nutrition: The use and misuse of fruit juice in pediatrics. Pediatrics 2001, 107:1210-3.

10. Dennison BA, Rockwell HL, Baker SL: Excess fruit juice consumption by preschool-aged children is associated with short stature and obesity. Pediatrics 1997, 99:15-22.

11. Epstein LH, Gordy CC, Raynor HA, Beddome M, Kilanowski CK, Paluch R: Increasing fruit and vegetable intake and decreasing fat and sugar intake in families at risk for childhood obesity. Obes Res 2001, 9:171-178.

12. Faith MS, Dennison BA, Edmunds LS, Stratton HH: Fruit juice intake predicts increased adiposity gain in children from low-income families: weight status-by-environment interaction. Pediatrics 2006, 118:2066-75.

13. Melgar-Quinonez HR, Kaiser LL: Relationship of child-feeding practices to overweight in low-income Mexican-American preschool-aged children. J Am Diet Assoc 2004, 104:1110-1119.

14. Newby PK, Peterson KE, Berkey CS, Leppert J, Willett WC, Colditz GA: Beverage consumption is not associated with changes in weight and body mass index among low-income preschool children in North Dakota. J Am Diet Assoc 2004, 104:1086-1094.

15. Nicklas TA, O'Neil CE, Kleinman R: Association between $100 \%$ juice consumption and nutrient intake and weight of children 2 to 11 years. Arch Pediatr Adolesc Med 2008, 162:557-565.

16. O'Neil CE, Nicklas TA, Kleinman R: Relationship between $100 \%$ juice consumption and nutrient intake and weight of adolescents. Am J Health Promot 2010, 24:231-7.

17. O'Connor TM, Yang SJ, Nicklas TA: Beverage intake among preschool children and its effect on weight status. Pediatrics 2006, 118:e1010-8

18. La Rowe TL, Moeller SM, Adams A: Beverage patterns, diet quality, and body mass index of US preschool and school-aged children. J Am Diet Assoc 2007, 107:1124-1133.

19. Forshee RA, Storey ML: Total beverage consumption and beverage choices among children and adolescents. Int J Food Sci Nutr 2003, 54:297-307.

20. O'Neil CE, Nicklas TA: A review of the relationship between $100 \%$ fruit juice consumption and weight in children and adolescents. Am J Lifestyle Med 2008, 2:315-354

21. Schulze MB, Manson JE, Ludwig DS, Colditz GA, Stampfer MJ, Willett WC, Hu FB: Sugar-sweetened beverages, weight gain, and incidence of type 2 diabetes in young and middle-aged women. JAMA 2004, 292:927-34.

22. Bes-Rastrollo M, Sanchez-Villegas A, Gomez-Gracia E, Martinez JA, Pajares RM, Martinez-Gonzalez MA: Predictors of weight gain in a Mediterranean cohort: the Seguimiento Universidad de Navarra Study 1. Am J Clin Nutr 2006, 83:362-70, quiz 94-5.

23. Akhtar-Danesh N, Dehghan M: Association between fruit juice consumption and self-reported body mass index among adult Canadians. J Hum Nutr Diet 2010, 23:162-8.

24. Rampersaud GC: A comparison of nutrient density scores for $100 \%$ fruit juices. J Food Sci 2007, 72:S261-6.

25. Carlsen M, Halvorsen B, Holte K, Bohn S, Dragland S, Sampson L, Willey C, Senoo H, Umezono Y, Sanada C, Barikmo I, Berhe N, Willett WC, Phillips KM, Jacobs DR Jr, Blomhoff R: The total antioxidant content of more than 3100 foods, beverages, spices, herbs and supplements used worldwide. Nutr J 2010, 9:3.

26. U.S. Department of Agriculture: 2010 Dietary Guidelines Advisory Committee Report [http://www.cnpp.usda.gov/dietaryguidelines.htm].

27. National Center for Health Statistics: About the National Health and Nutrition Examination Survey [http://www.cdc.gov/nchs/nhanes/about_nhanes.htm].

28. National Center for Health Statistics: NHANES 1999-2000 Public Data Release File Documentation [http://www.cdc.gov/nchs/data/nhanes/nhanes_99_00/ general_data_release_doc.pdf].

29. Moshfegh AJ, Rhodes DG, Baer DJ, Murayi T, Clemens JC, Rumpler WW, Paul DR, Sebastian RS, Kuczynski K, Ingwersen LA, Staples RC, Cleveland LE: The US Department of Agriculture Automated Multiple-Pass Method reduces bias in the collection of energy intakes. Am J Clin Nutr 2008, 88:324-32.

30. Blanton CA, Moshfegh AJ, Baer DJ, Kretsch MJ: The USDA Automated Multiple-Pass Method accurately estimates group total energy and nutrient intake. J Nutr 2006, 136:2594-9.

31. National Center for Health Statistics: The NHANES 2002 MEC In-Person Dietary Interviewers Procedures Manual [http://www.cdc.gov/nchs/data/nhanes/ nhanes_01_02/dietary_year_3.pdf].
32. U.S. Department of Agriculture, Agricultural Research Service: USDA Food and Nutrient Database for Dietary Studies, 2.0. Beltsville, MD 2006 [http:// www.ars.usda.gov/Services/docs.htm?docid=12083].

33. U.S. Department of Agriculture, Agricultural Research Service: USDA Food and Nutrient Database for Dietary Studies, 3.0. Beltsville, MD 2008 [http:// www.ars.usda.gov/Services/docs.htm?docid=17031].

34. Federal Food and Drug Administration: Part 101 - Food Labeling, Subpart B Sec. 101.3. Percentage of juice declaration for foods purporting to be beverages that contain fruit or vegetable juice [21CFR101.30] [http://www.accessdata.fda. gov/scripts/cdrh/cfdocs/cffrr/CFRSearch.cfm?fr=101.30].

35. Guenther PM, Reedy J, Krebs-Smith SM, Reeve BB, Basiotis PP: Development and evaluation of the Healthy Eating Index-2005: Technical report. Center for Nutrition Policy and Promotion, U.S. Department of Agriculture; 2007 [http://www.cnpp.usda.gov/Publications/ HEl/HEl-2005/HEl-2005TechnicalReport.pdf].

36. National Cancer Institute: Healthy Eating Index - 2005 Tools for Researchers. Sample SAS code to create HEl-2005 scores [http://riskfactor.cancer.gov/tools/ hei/tools.html]

37. National Cancer Institute: Usual Dietary Intakes: SAS Macros for the NCI Method [http://riskfactor.cancer.gov/diet/usualintakes/macros.html].

38. National Center for Health Statistics: Analytic and Reporting Guidelines. The National Health and Nutrition Examination Survey (NHANES) [http://www.cdc. gov/nchs/data/nhanes/nhanes_03_04/ nhanes_analytic_guidelines_dec_2005.pdf].

39. U.S. Department of Agriculture, Food and Nutrition Service: Program Data. WIC Program, National Level Annual Summary: FY 1974-2009 [http://www.fns. usda.gov/pd/wicmain.htm]

40. U.S. Department of Agriculture, Food \& Nutrition Service: WIC Food Packages -Regulatory Requirements for WIC-Eligible Foods [http://www.fns. usda.gov/wic/benefitsandservices/foodpkgregs.HTM\#JUICE_(Women_an d Children)].

41. Condon EM, Crepinsek MK, Fox MK: School meals: types of foods offered to and consumed by children at lunch and breakfast. J Am Diet Assoc 2009, 109:567-78.

42. U.S. Department of Agriculture, Food \& Nutrition Service: Food Buying Guide for Child Nutrition Programs. Section 2: Vegetables/Fruits [http://teamnutrition. usda.gov/Resources/FBG_Section_2-VegFruits.pdf].

43. Deshmukh-Taskar PR, Nicklas TA, O'Neil CE, Keast DR, Radcliffe JD, Cho S: The relationship of breakfast skipping and type of breakfast consumption with nutrient intake and weight status in children and adolescents: the National Health and Nutrition Examination Survey 1999-2006. J Am Diet Assoc 2010, 110:869-78.

44. Kakarala M, Keast DR, Hoerr S: Schoolchildren's consumption of competitive foods and beverages, excluding à la carte. J Sch Health 2010, 80:429-35.

45. Nelson MC, Neumark-Sztainer D, Hannan PJ, Story M: Five-year longitudinal and secular shifts in adolescent beverage intake: findings from project EAT (Eating Among Teens)-II. J Am Diet Assoc 2009, 109:308-12.

46. Institute of Medicine: Dietary Reference Intakes for Energy, Carbohydrate, Fiber, Fat, Fatty Acids, Cholesterol, Protein, and Amino Acids (Macronutrients) Washington DC: The National Academies Press; 2005.

47. US Department of Agriculture: 2005 Dietary Guidelines for Americans [http:// www.health.gov/dietaryguidelines/dga2005/document/default.htm].

48. Freedman LS, Guenther PM, Krebs-Smith SM, Kott PS: A population's mean Healthy Eating Index-2005 scores are best estimated by the score of the population ratio when one 24-hour recall is available. J Nutr 2008, 138:1725-9.

49. Freedman LS, Guenther PM, Krebs-Smith SM, Dodd KW, Midthune D: A population's distribution of Healthy Eating Index-2005 component scores can be estimated when more than one 24 -hour recall is available. J Nutr 2010, 140:1529-34.

50. Guenther PM, Juan WY, Lino M, Hiza HA, Fungwe T, Lucas R: Diet quality of low-income and higher income Americans in 2003-04 as measured by the Healthy Eating Index-2005: Nutrition Insight 42. US Department of Agriculture, Center for Nutrition Policy and Promotion; 2008 [http://www. cnpp.usda.gov/Publications/Nutritionlnsights/Insight42.pdf].

51. Casagrande SS, Wang Y, Anderson C, Gary TL: Have Americans increased their fruit and vegetable intake? The trends between 1988 and 2002. Am J Prev Med 2007, 32:257-263.

52. Produce for Better Health. [http://www.fruitsandveggiesmorematters.org]. 
53. Tanasescu M, Ferris AM, Himmelgreen DA, Rodriguez N, Perez-Escamilla R: Biobehavioral factors are associated with obesity in Puerto Rican children. J Nutr 2000, 130:1734-42.

54. Reedy J, Krebs-Smith SM: Dietary sources of energy, solid fats, and added sugars among children and adolescents in the United States. J Am Diet Assoc 2010, 110:1477-84.

55. Basch CE, Shea S, Arliss R, Contento IR, Rips J, Gutin B, Irigoyen M, Zybert P. Validation of mothers' reports of dietary intake by four to seven yearold children. Am J Public Health 1990, 80:1314-1317.

56. Baranowski T, Sprague D, Baranowski JH, Harrison JA: Accuracy of maternal dietary recall for preschool children. J Am Diet Assoc 1991, 91:669-674.

57. Schoeller DA: How accurate is self-reported dietary energy intake? Nutr Rev 1990, 48:373-379.

doi:10.1186/1475-2891-10-17

Cite this article as: O'Neil et al: Diet quality is positively associated with $100 \%$ fruit juice consumption in children and adults in the United States: NHANES 2003-2006. Nutrition Journal 2011 10:17.

\section{Submit your next manuscript to BioMed Central} and take full advantage of:

- Convenient online submission

- Thorough peer review

- No space constraints or color figure charges

- Immediate publication on acceptance

- Inclusion in PubMed, CAS, Scopus and Google Scholar

- Research which is freely available for redistribution

Submit your manuscript at www.biomedcentral.com/submit
C Biomed Central 